\title{
Improving sustainable use of genetic resources in biodiversity archives
}

\author{
E J Tuschhoff ${ }^{\text {Corresp., 1, 2, } 3}$, Carl R Hutter ${ }^{2,3}$, Richard E Glor ${ }^{2,3}$ \\ ${ }^{1}$ Department of Ecology and Evolutionary Biology, University of Arizona, Tucson, Arizona, United States of America \\ 2 Department of Ecology and Evolutionary Biology, University of Kansas, Lawrence, Kansas, United States of America \\ 3 University of Kansas Biodiversity Institute, Lawrence, Kansas, United States of America \\ Corresponding Author: E J Tuschhoff \\ Email address: etuschhoff@email.arizona.edu
}

Tissue sample databases housed in biodiversity archives represent a vast trove of genetic resources, and these tissues are often destructively subsampled and provided to researchers for DNA extractions and subsequent sequencing. While obtaining a sufficient quantity of DNA for downstream applications is vital for these researchers, it is also important to preserve tissue resources for future use given that the original material is destructively and consumptively sampled with each use. It is therefore necessary to develop standardized tissue subsampling and loaning procedures to ensure that tissues are being used efficiently. In this study, we specifically focus on the efficiency of DNA extraction methods by using anuran liver and muscle tissues maintained at a biodiversity archive. We conducted a series of experiments to test whether current practices involving coarse visual assessments of tissue size are effective, how tissue mass correlates with DNA yield and concentration, and whether the amount of DNA recovered is correlated with sample age. We found that tissue samples between $2 \mathrm{mg}$ and $8 \mathrm{mg}$ resulted in the most efficient extractions, with tissues at the lower end of this range providing more DNA per unit mass and tissues at the higher end of this range providing more total DNA. Additionally, we found no correlation between tissue age and DNA yield. Because we find that even very small tissue subsamples tend to yield far more DNA than is required by researchers for modern sequencing applications (including whole genome shotgun sequencing), we recommend that biodiversity archives consider dramatically improving sustainable use of their archived material by providing researchers with set quantities of extracted DNA rather than with the subsampled tissues themselves. 
1 IMPROVING SUSTAINABLE USE OF GENETIC

2 RESOURCES IN BIODIVERSITY ARCHIVES

3

4

5

6

7

\author{
E J. Tuschhoff ${ }^{1,2,3}$, Carl R. Hutter ${ }^{1,2}$, Richard E. Glor ${ }^{1,2}$ \\ ${ }^{1}$ Department of Ecology and Evolutionary Biology, University of Kansas, Lawrence, Kansas, \\ United States of America \\ 2 University of Kansas Biodiversity Institute, Lawrence, Kansas, United States of America \\ ${ }^{3}$ Department of Ecology and Evolutionary Biology, University of Arizona, Tucson, Arizona, \\ United States of America
}

Corresponding Author:

E Tuschhoff $\mathrm{f}^{1,2,3}$

BioSciences West room 310, 1041 E Lowell St, Tucson, Arizona, 85721, United States of

America

Email address: etuschhoff@email.arizona.edu

\title{
Abstract
}

Tissue sample databases housed in biodiversity archives represent a vast trove of genetic resources, and these tissues are often destructively subsampled and provided to researchers for DNA extractions and subsequent sequencing. While obtaining a sufficient quantity of DNA for downstream applications is vital for these researchers, it is also important to preserve tissue resources for future use given that the original material is destructively and consumptively sampled with each use. It is therefore necessary to develop standardized tissue subsampling and loaning procedures to ensure that tissues are being used efficiently. In this study, we specifically focus on the efficiency of DNA extraction methods by using anuran liver and muscle tissues maintained at a biodiversity archive. We conducted a series of experiments to test whether current practices involving coarse visual assessments of tissue size are effective, how tissue mass correlates with DNA yield and concentration, and whether the amount of DNA recovered is correlated with sample age. We found that tissue samples between $2 \mathrm{mg}$ and $8 \mathrm{mg}$ resulted in the most efficient extractions, with tissues at the lower end of this range providing more DNA per unit mass and tissues at the higher end of this range providing more total DNA. Additionally, we found no correlation between tissue age and DNA yield. Because we find that even very small tissue subsamples tend to yield far more DNA than is required by researchers for modern sequencing applications (including whole genome shotgun sequencing), we recommend that biodiversity archives consider dramatically improving sustainable use of their archived material by providing researchers with set quantities of extracted DNA rather than with the subsampled tissues themselves. 


\section{Introduction}

41 Genetic resources archived in biodiversity collections are critically important for scientific

42

43

44

45

46

47

48

49

50

51

52

53

54

55

56

57

58

59

60

61

62

63

64

65

66

67

68

69

70

71

72

73

74

75

76

77

78 research because they permit immediate access to large numbers of samples obtained across taxa, time and space, including samples that would be difficult or even impossible to obtain today (Droege et al. 2014, Burrell et al. 2015, Schaffer et al. 2017). Increasing reliance on archived genetic resources by a growing community of researchers, however, presents a significant challenge because current methods for sharing genetic resources are not sustainable; in most cases, researchers requesting access to genetic resources are provided with a piece of tissue that is consumptively subsampled from a permanently archived resource (Zimkus and Ford 2014). Researchers then destroy this subsample during the course of DNA extraction, use the DNA that is required for their research and typically discard any remaining material. As a result, every request to use genetic resources results in depletion of samples that, left unchecked, will result in complete sample exhaustion and permanent loss of an irreplaceable resource. Because some tissues are present in very small quantities, some genetic resources can only be provided to one or a few researchers before an irreplaceable resource is lost forever. This issue becomes especially pressing when one considers the current extinction crises and increasingly strict regulations for scientific collecting that may prevent samples being replenished from wild specimens (Stuart et al. 2004, Watanabe 2015). As a result, it is important to develop protocols that improve sustainable use of these resources.

Because the vast majority of requests to use archived genetic resources involve efforts to sequence DNA, protocols for DNA extraction from archival tissues are an obvious focal point for optimization aimed at improving sustainability of current practices. Most biodiversity collections aim to provide researchers requesting access to genetic material with enough tissue to conduct two DNA extractions (Zimkus and Ford 2014), but collections staff and researchers are often unaware of how much tissue is optimal for extraction because few studies have investigated how sample age, preservation method, extraction protocol, type of tissue, and subsample size are related to the quantity, concentration, and quality of extracted DNA (but see Reineke 1998, Drabkova 2002, Guo 2009, Sawyer 2012, Choi 2015, Schiebelhut 2016, and Abdel-Latif 2017). Even parameters that are known to impact extraction success are rarely quantified when biodiversity collections fulfill requests for access to genetic material. For example, tissue mass is known to be strongly correlated with extraction success (Hykin et al. 2015) and has been shown to be correlated with extracted DNA concentration (Reineke et al. 1998, Choi et al. 2015)but collections staff and researchers generally use a coarse visual estimate when removing tissue subsamples and rarely obtain quantitative size or mass data. It is not currently common practice to standardize tissue mass prior to DNA extractions (Wilcox et al. 2002, Aguirre-Peñafiel et al. 2014, Naccarato et al. 2015) or to report masses if they were standardized (Kayes et al. 2013) except in experiments to compare various protocols or methods (Drabkova et al. 2002, Guo et al. 2009, Abdel-Latif and Osman 2017, Yalcinkaya et al. 2017). In 
79 publications, researchers tend to qualitatively report the amount of starting material with phrases

80 such "two small pieces" or "usually minute" (Hajibabaei et al. 2005, Jaksch et al. 2016).

81

82

83

84

85

86

87

88

89

90

91

92

93

94

95

96

97

98

99

100

101

102

103

104

105

106

107

108

109

110

111

112

113

114

115

116

117

The goal of the present study is to develop guidelines for more sustainable use of genetic resources in biodiversity collections, with a focus on determining the optimal amount of tissue for DNA extraction from amphibian tissue samples. In our first experiment we test whether the type of coarse visual estimates of tissue mass or size that are used by most collections staff who fulfill requests for access to genetic resources are capable of consistently yielding sufficient DNA for modern downstream sequencing applications. In our second experiment, we identify the tissue masses that result in the most efficient use of archived samples by conducting controlled extractions across a range of samples with known masses. In our third experiment, we test consistency of extraction success across replicate subsamples of a mass that appears to optimize yield while minimizing depletion of the archived samples during a single extraction. In our fourth and final experiment, we test whether our protocol is suitable for samples archived over a 25-year interval from 1984 (around the time collections started accumulating sample preserved specifically for use in molecular genetic studies) until 2001. Given the nature of natural history collections, it is probable that researchers will need to work with tissues of a variety of ages. Previous studies of bone and plant tissues have not recovered a significant correlation between DNA yield and tissue age (Sawyer et al. 2012, Choi et al. 2015), and, to our knowledge, previous published studies have not tested the correlation between age and total DNA yields using cryogenically preserved soft tissues from vertebrates.

. However, one study of herpetological specimens found a significant decrease in recovered sequence length as tissue age increased (Chambers and Hebert 2016).

\section{Materials \& Methods}

\section{Sampling}

We conducted all our experiments on amphibian tissues samples from the herpetological collection at the University of Kansas Biodiversity Institute. With more than 40,000 tissue samples in cryogenic storage, this collection is among the largest archives of its kind. This collection is also widely used by the scientific community, with more than 75 requests for access to genetic resources by the scientific community resulting in subsampling of more than 1,100 archived samples over the past five years. We focused on liver and muscle tissue because these tissues are the most abundant in biodiversity archives and are usually the standard tissue types collected in the field. Tissues were initially preserved using one of two strategies: immersion in high concentration ethanol or flash freezing in liquid nitrogen. Subsequent to initial preservation, samples were stored in a cryogenic facility, either in mechanical ultra-cold freezers at $-80^{\circ} \mathrm{C}$ (experiments 1-3) or liquid nitrogen cooled dewars at $-180^{\circ} \mathrm{C}$ (experiment 4 ).

\section{Tissue Extraction Protocol}


118 The majority of the tissues used in this experiment were stored in ethanol solution. Tissues that 119 had been flash frozen and were not stored in ethanol solution were transferred to a $95 \%$ ethanol

120

121

122

123

124

125

126

127

128

129

130

131

132

133

134

135

136

137

138

139

140

141

142

143

144

145

146

147

148

149

150

151

152

153

154

155

156

157 solution and allowed to thaw to $-80^{\circ} \mathrm{C}$ such that all tissues were under the same conditions at the time of massing. All tissues were next removed from ethanol and the ethanol was allowed to evaporate for up to two minutes to limit the contribution of ethanol to inferred tissue mass. Each tissue was subsampled with a sterile razor blade until the mass was within $0.5 \mathrm{mg}$ of the target mass as measured by a Mettler Toledo XS105DU analytical balance scale (in 8 cases, masses more than $.5 \mathrm{mg}$ under the target mass were used because there was not adequate tissue remaining for the full amount, see additional details below). Tissues were then placed in a solution of $10 \mu \mathrm{L}$ protein kinase and $190 \mu \mathrm{L}$ lysis buffer and incubated at $55^{\circ} \mathrm{C}$ for approximately 24 hours (several of the larger masses required longer incubation times for complete tissue digestion as determined by the absence of solid tissue pieces in the solution).

Tissue solutions were vortexed once at the start of the incubation period for ten seconds and one to three times at the end of the incubation period depending on the level of tissue digestion.

The extractions in this experiment were performed using the Promega Maxwell RSC Instrument (Promega Corporation, Maddison, Wisconsin, USA). The Maxwell RSC uses paramagnetic particles along with magnetic plungers to lyse and capture DNA along with specialized reagents provided in single use cartridges (Kephart et al. 2006). Aside from lysis and transfer to a sterile Eppendorf tube for quantification and storage, the extraction process is entirely automated and occurs inside the instrument. This method was chosen for our experiments for three reasons, and in spite of the fact that the method has relatively high costs both in terms of initial investment in the machine ( $>\$ 20,000)$ and for individual extractions ( $\sim \$ 8$ per cartridge) as of June 26, 2019. First, a recent comparative analysis of commonly used extraction protocols found that the Promega paramagnetic particle method results in particularly high DNA yields, high sample efficacy (measured in the success of PCR), and low error (Schiebelhut et al. 2016). Secondly, this automated extraction method allows for a high degree of uniformity across multiple trials and reduces the human error inherent in manual protocols. Finally, third, the Promega RSC instrument relies on sterile individual use cartridges, a drip-free protocol, and includes an automated UV sterilization of internal components following each extraction, which collectively minimize the potential for contamination.

In our study, we used the Promega blood DNA purification kit (Promega product ID: AS1010). We followed the manufacturer's procedures during the extraction except that elution buffer volume was doubled to $100 \mu \mathrm{L}$ because at lower volumes the quantity of DNA could not be read by a fluorometer as DNA concentrations were too high. After extraction was completed, quantifications were performed using a Promega Quantus fluorometer.

Experiment 1: Testing the effectiveness of the "eyeball" method for obtaining tissues appropriate for extraction 
158 We first conducted a preliminary experiment to determine if coarse visual assessment of tissue

159

160

161

162

163

164

165

166

167

168

169

170

171

172

173

174

175

176

177

178

179

180

181

182

183

184

185

186

187

188

189

190

191

192

193

194

195

196

197

mass (i.e., the "eyeball" approach to tissue quantification used by most biodiversity collections staff) is capable of sampling tissues that result in consistent DNA yield which are sufficient for modern downstream DNA sequencing applications. The concentration and amount of DNA required for sequencing depends on the sequencing method used, ranging from less than $10 \mathrm{ng}$ of DNA for Sanger sequencing a single DNA fragment to $500 \mathrm{ng}$ for Illumina Truseq-style library preparation (Hutter et al. 2019) to over $1000 \mathrm{ng}$ for high coverage sequencing of an entire vertebrate genome via the Illumina platform (Arbor Biosciences). Because $1000 \mathrm{ng}$ is at the high end of the amount used for standard sequencing methods applied to typical vertebrate genomes (including whole genome sequencing and popular methods such as RADseq and probe capture), we used this amount as our threshold for establishing extraction success.

For this experiment, two experienced scientists (Drs. Carl Hutter and Shea Lambert) attempted to consistently subsample tissues with a mass considered sufficiently large for DNA extraction based on prior experience. Tissue subsamples obtained in this manner were then weighed prior to extraction and quantification. Although the researchers knew that their subsamples were being massed, they were asked to subsample per their normal procedures and were not given any feedback about the masses of their samples. Following extraction, we tested whether each sample passed our $1000 \mathrm{ng}$ minimum threshold for successful extraction. We also tested the basic prediction that tissue mass is correlated with DNA yield using a Pearson's correlation test. Finally, we tested reliability of "eyeball" estimates of tissue mass by estimating variance in both the mass and DNA yield of resulting subsamples.

\section{Experiment 2: Identification of optimal tissue mass for effective and efficient extraction}

Our second experiment focused on identifying the optimal tissue masses for DNA extraction, which we define here as the masses that results in high DNA yield per unit tissue mass and high overall DNA yield. For this experiment, we conducted a total of 123 extractions from tissue samples of eight different masses: $1 \mathrm{mg}, 2 \mathrm{mg}, 4 \mathrm{mg}, 8 \mathrm{mg}, 10 \mathrm{mg}, 12 \mathrm{mg}, 14 \mathrm{mg}, 16 \mathrm{mg}$, and 20 $\mathrm{mg}$. This range was chosen because $1 \mathrm{mg}$ was determined to be the smallest mass that could be reliably manipulated by the experimenter and $20 \mathrm{mg}$ was the maximum mass recommended by our extraction protocol. Tissues were assigned to a sample mass if they were within $.5 \mathrm{mg}$ of the target mass. In eight cases, there was insufficient tissue to subsample the desired tissue mass and the actual subsample mass was therefore more than $.5 \mathrm{mg}$ outside the targeted masses. In these instances, tissues were placed in the category to which they were closest, and all were less than $1.2 \mathrm{mg}$ from the target mass. Tissue samples for this experiment were 24 liver tissue samples obtained from Malagasy frogs in 2016 which were all from the family Mantellidae and one sample from Ranidae. Each tissue was sampled 4-12 times at various masses depending on the total tissue mass of the original sample. All of the samples used in this experiment were initially preserved in ethanol and stored at room temperature for a period of several weeks and up to two months before being transferred to cryogenic storage in either a mechanical ultracold freezer (-

Peer) reviewing PDF | (2019:08:40184:2:0:NEW 25 Nov 2019) 
198

199

200

201

202

203

204

205

206

207

208

209

210

211

212

213

214

215

216

217

218

219

220

221

222

223

224

225

226

227

228

229

230

231

232

233

234

235

236

237

$80 \mathrm{C})$ or a liquid nitrogen cooled dewar (-180C). In each extraction run, 4 tissues each with 4 subsamples were extracted for a total of 16 extractions. The data was analyzed using a least squares regression to fit a trend line.

\section{Experiment 3: Consistency of extraction yield at an optimal mass}

Our third experiment assessed the consistency of extraction yield from tissue subsamples at a sample mass identified in Experiment 2 that results in both high DNA yield per unit mass and high overall DNA yield without involving masses so large as to permit only one or two extractions from small tissue samples. Because this experiment required 4 subsamples of $8 \mathrm{mg}$ from each tissue, large samples such as those from Mantellidae were needed. Six Mantellidae tissues were sampled for a total of 32 subsamples ( 2 tissues were used twice due to a lack of suitable tissues). In each extraction run, 4 tissues each with 4 subsamples were extracted for a total of 16 extractions.

\section{Experiment 4: Impact of age on extractions using the optimal mass}

The fourth experiment was conducted using 44 historical anuran samples including both ethanol preserved and flash frozen samples. These samples belonged to several different frog families: Bufonidae (3 samples), Dendrobatidae (10), Hylidae (17), Leptodactylidae (11), and 3 from unknown families. These tissues ranged in collection date from 1984 to 2001 and included both liver and muscle tissue. We sampled, extracted, and quantified $8 \mathrm{mg}$ of each tissue using the same procedure as described above. Data was analyzed using a Pearson's correlation test.

\section{Results}

Experiment 1: Testing the effectiveness of the "eyeball" method for obtaining tissues appropriate for extraction

We found that coarse visual estimates of tissue subsamples resulted in a wide range of resulting tissue masses (0.65-14.93 mg). The mean mass was $3.33 \mathrm{mg}$ with a standard deviation of 3.32 mg. All but the smallest of the tissues extracted during this experiment resulted in DNA yields that exceeded our $1000 \mathrm{ng}$ threshold. We also found that DNA yield is significantly positively correlated with original tissue mass (Pearson correlation test: $\mathrm{t}=5.2299, \mathrm{r}=0.7600, \mathrm{df}=20$, $\mathrm{p}$ value $<0.001$, Figure 1). Additionally, when the three samples with the greatest mass were removed from the analysis, DNA yield was still found to be significantly positively correlated with tissue mass (Pearson correlation test: $\mathrm{t}=2.3112, \mathrm{r}=0.4890, \mathrm{df}=17, \mathrm{p}$-value $=0.0336$, Figure $1)$.

\section{Experiment 2: Identification of optimal tissue mass for effective and efficient extraction} In the second experiment, we recovered a non-linear relationship between tissue mass and both concentration and total DNA yield (Figure 2). The smallest tissue subsamples (1 mg, $2 \mathrm{mg}$, and $4 \mathrm{mg}$ ) yielded a mean of $76.8 \mathrm{ng} / \mu \mathrm{L}$ of DNA. The intermediate tissues $(8 \mathrm{mg}, 10 \mathrm{mg}$, and $12 \mathrm{mg}$ ) 
238

239

240

241

242

243

244

245

246

247

248

249

250

251

252

253

254

255

256

257

258

259

260

261

262

263

264

265

266

267

268

269

270

271

272

273

274

275

276

277

yielded a mean of $123.5 \mathrm{ng} / \mu \mathrm{L}$ of DNA. The largest tissues (14 mg, $16 \mathrm{mg}$, and $20 \mathrm{mg}$ ) yielded a mean of $144.6 \mathrm{ng} / \mu \mathrm{L}$ of DNA. These data were best fit by the natural $\log$ equation) $\mathrm{y}=3317.2 * \ln (\mathrm{x})+5030.3\left(\mathrm{R}^{2}=0.29\right.$, $\mathrm{p}$-value $\left.<0.0001\right)$. The relationship between tissue mass and DNA concentration shows a gradual decrease in the DNA gained per mg of tissue as the total tissue mass increases. While the natural log function does not have an asymptote, it may reach a point where the extra DNA that could be obtained is so little that it is not worth the additional destructive use of limited tissue resources. The intermediate and large tissue masses ( $8 \mathrm{mg}$ and higher) also tend to result in higher overall DNA yields. Although these masses tend to result in both higher DNA concentrations and higher overall DNA yields, yield per unit mass is greatest for the small tissues, with a mean of $3444.5 \mathrm{ng} \mathrm{DNA} / \mathrm{mg}$ tissue, as compared to $1288.7 \mathrm{ng}$ $\mathrm{DNA} / \mathrm{mg}$ tissue for intermediate masses and $922.6 \mathrm{ng} \mathrm{DNA} / \mathrm{mg}$ tissue for large masses. These data were best fit by the natural log equation $y=-1337 * \ln (x)+4470.3\left(R^{2}=0.55\right.$, p-value $\left.<0.0001\right)$.

\section{Experiment 3: Consistency of extraction yield at optimal mass}

The third experiment further analyzed the precision of using $8 \mathrm{mg}$ of tissue. We analyzed 28 mantellid tissue samples over 32 extractions. One tissue and its four corresponding subsamples were discarded from this analysis resulting in DNA concentrations that were significantly lower from those for all other tissues (Tukey Honest Significant Differences, p-values 2.07E-7 to 2.96E-2); we suspect that this tissue was degraded and does not contain sufficient quantities of DNA to result in useful yields following standard DNA extraction methods. The mean DNA concentration from samples extracted during this experiment was $133.75 \mathrm{ng} / \mu \mathrm{L}$ with a mean yield of 13,375 ng of DNA. The mean standard deviation of DNA concentration among subsamples of the same tissue was $19.12 \mathrm{ng}$ and the mean range was $41.86 \mathrm{ng}$.

\section{Experiment 4: Impact of age on extractions using the optimal mass}

The fourth experiment tested whether the age of tissue samples impacts the expected relationship between sample mass and DNA yield for 44 archival tissues. The average mass of tissue used in this experiment was $7.86 \mathrm{mg}$ with an average yield of $104.56 \mathrm{ng} / \mu \mathrm{L}$ of DNA. This experiment found no correlation (Pearson correlation: $r=-0.06$, $p$-value $=0.6904$ ) between the age of a tissue sample and the concentration of DNA yielded (Figure 3).

\section{Discussion}

The goal of our study was to develop guidelines for sustainable use of tissue samples archived in biodiversity collections that are destructively subsampled for DNA extraction. We found that current tissue sampling methods involving coarse visual assessment of tissue size generally yield sufficient DNA for modern downstream applications. However, the actual yield from samples obtained via the "eyeball" method is highly variable, and, because tissue mass is correlated with DNA yield, massing tissues prior to extraction will increase consistency and efficiency.

Intermediate and large tissue masses yielded comparable concentrations of DNA, but small 
278 tissue masses had the greatest DNA yield per unit mass. Additionally, sample age was not 279 correlated with DNA yield.

280

281

282

283

284

285

286

287

288

289

290

291

292

293

294

295

296

297

298

299

300

301

302

303

304

305

306

307

308

309

310

311

312

313

314

315

316

317
In our first experiment, we showed that the methods currently used by many biodiversity archives, which involve coarse visual estimates of tissue amounts that are considered sufficient for DNA extraction based on prior experience, generally yield more than enough DNA for most modern downstream applications, including whole genome sequencing. However, we also found that tissues subsampled in this manner do not produce consistent amounts of DNA because they encompassed a wide range of masses $(0.64 \mathrm{mg}-14.93 \mathrm{mg})$, and DNA yield is strongly correlated with mass. Overall this experiment suggests that use of archived tissue samples would be more efficient if tissues were massed prior to distribution. Of course, this strategy does not come without costs. First, quantification of tissue subsample mass requires a significant additional investment in handling time and access to an expensive analytical balance capable of accurately weighing samples in the 1-20 mg range. As with any increase in handling time, this approach may also result in accelerated degradation of archived samples. However, the benefits of standardization may outweigh these costs, particularly in the case of samples that are only available in limited quantities.

Generally speaking, standardization of tissue masses provided to researchers for extraction will improve the process of intercollection tissue loans because loanees will be sure to receive a quantity of tissue that will result in the required quantity of DNA. The need for an overall standard tissue loan procedure has been previously highlighted (Droege et al. 2014) and we believe that, given the strong correlation between tissue mass and DNA yield, standardization of tissue mass could be one important step in this direction. Given the varying specimens housed in different tissue collections, researchers often require tissue loans from other institutions in order to complete their work. It is expected that these tissues will yield sufficient DNA for experimentation, but often collections do not wish to part with the last pieces of a tissue sample. A survey of 45 institutions with genetic resource holdings revealed that none of the $93 \%$ of institutions that offered loans sent loanees the entire tissue sample, and amount of tissue sent varied between institutions (Zimkus and Ford 2014). For example, 25\% of collections reported sending enough tissue for two extractions and $9 \%$ sent enough for three extractions, but only $21 \%$ of institutions quantified tissue sent (either by volume or mass). The loan procedures posted on the websites of seven major herpetological collections in the United States (Berkeley Museum of Vertebrate Zoology, California Academy of Sciences, Museum of Comparative Zoology at Harvard University, Smithsonian Museum of Natural History, University of Florida, University of Kansas, and University of Texas) revealed that these collections provided detailed and welldefined loan procedures for whole animal specimens, but generally provide little detail on procedures for providing genetic resources. Correspondence with collections managers at these institutions revealed a variety of approaches and techniques for determining the amount of tissue to provide researchers requesting access to genetic resources, including qualitative visual

Peer] reviewing PDF | (2019:08:40184:2:0:NEW 25 Nov 2019) 
318

319

320

321

322

323

324

325

326

327

328

329

330

331

332

333

334

335

336

337

338

339

340

341

342

343

344

345

346

347

348

349

350

351

352

353

354

355

356

357

assessment, tissue volume, the minimum tissue required for the proposed project, and approximate mass (Huddleston, Scheinberg, Spencer, Zimkus; personal communications March 2019). Standardization of tissue masses would allow loanees to receive a previously agreed upon tissue mass that has been shown to yield appropriate amounts of DNA for their proposed downstream applications, while loaners can improve sustainable use of their tissue collections by only loaning the required amount of tissue.

In our second experiment, we recovered a non-linear increase in DNA concentration and total yield with increasing tissue mass, with the smallest masses resulting in considerably lower concentrations and yields than intermediate or large tissue masses. However, the yield per starting quantity mass of tissue, a measure of how efficiently we are recovering DNA from the original tissue sample, is highest at the smallest masses and declines dramatically with tissue sizes greater than $2 \mathrm{mg}$. For this reason, the decision about which mass is optimal for extraction will depend on a range of factors including the desired application and the total amount of tissue available. For samples available in only very limited quantities, extractions using only $2 \mathrm{mg}$ of tissue will often be ideal because they generally result in sufficient DNA for most downstream sequencing applications while optimizing efficient use of the available material by maximizing DNA yield per unit tissue used (Figure 2). In cases where larger initial tissue samples are available, it may be preferable to use somewhat larger tissue masses for extraction because masses of $8 \mathrm{~g}$ and larger tend to produce considerably higher DNA concentrations and overall yields than small starting masses. In most cases, a single extraction of a larger tissue that produces somewhat lower yields per unit tissue mass than smaller masses will generally be preferable to repeated extractions of smaller samples due to the significant increases in handling time and other expenses associated with extraction. We recommend subsampling more than $2 \mathrm{mg}$ of tissue when removing samples from biodiversity archives for DNA extraction, depending on the amount of material available. Of course, the optimal tissue mass for DNA extraction will depend on the extraction method being utilized and also the intended downstream applications. For this reason, our results are specific to use of the Promega Maxwell platform. Additional work is required to determine the optimal tissue mass to subsample when other extraction methods are being employed. However, it is likely that all these methods will exhibit increased concentration and yield with tissue masses that are larger than the minimum that can be manipulated.

Our fourth experiment suggests that concentration and yield from samples obtained over a 25year interval are not significantly correlated with age, reflecting previous findings that extraction quality is not correlated with age (Sawyer et al. 2012, Choi et al. 2015). This suggests that the same masses identified as being ideal for extraction of recent samples are also appropriate for historical samples. However, we did not evaluate other important factors influenced by age such as fragmentation, which might have similar yields with increasing age, but higher fragmentation. 
358 We primarily focused on tissue types that are most commonly housed in biodiversity archives

359

360

361

362

363

364

365

366

367

368

369

370

371

372

373

374

375

376

\section{Conclusions}

378

379

380

381

382

383

384

385

386

387

388

389

390

391

392

393

394

395

396

397 destructively sampled.

and used for extractions. We therefore did not analyze several other sources of genetic material in natural history collections, namely formalin-fixed specimens and tissue samples treated with RNAlater. Previous work has attempted to extract high quality DNA from formalin-fixed specimens with varying results (Hykin et al. 2015, Jaksch et al. 2016). Because the procedures used for these types of extractions are more involved and less often used, we chose not to include any formalin-fixed tissue subsamples in our study but recommend repeating our study with these specimens once extractions procedures are better developed. Conversely, specimens treated with RNAlater are often deliberately collected fresh from the field for a specific hypothesis. These specimens are often used for RNA-Seq applications to assess variation in gene expression in different tissue types (Wang et al. 2009), but are increasingly used for DNA work as well. These extractions were not included in this study due to the lack of these tissues in the University of Kansas herpetological collection and also because RNA extraction protocols have many more variables to consider (e.g. time to freezing, freezing temperature, amount of RNAlater used, freeze-thaw cycles). Further research is needed to determine if the results of our study also apply to RNAlater treated tissue samples.

Our experiments analyzed current practices in tissue subsampling and DNA extraction in biodiversity collections. We found that extractions using 2-8 $\mathrm{mg}$ of tissue were the most efficient and did not recover a strong correlation between DNA yield and tissue age. Two specific recommendations for improving sustainable use of genetic resources in biodiversity archives emerge from our study. Our first recommendation could be achieved with relatively minor adjustments to existing loan procedures while the second would require a dramatic change in how biodiversity archives provide researchers with access to genetic resources.

First, we discussed in detail the potential value of providing researchers with tissue samples of known mass. By standardizing the mass of tissues provided as gifts to researchers, the loaning institution be will be better able to ensure that researchers are provided with sufficient material while also being able to make more informed decisions about how limited resources are

Our second recommendation derives from our finding that even very small quantities of tissue often produce far more DNA than is required for most applications. For example, we found that tissues subsamples weighing $8 \mathrm{mg}$ tend to yield more than 13 times the amount of DNA that is required even for whole genome shotgun sequencing. In most cases, excess DNA obtained by researchers who receive tissue loans is discarded. Even in cases where institutions are capable of archiving extracted DNA and request return of unused material, this rarely happens in practice

Peer] reviewing PDF | (2019:08:40184:2:0:NEW 25 Nov 2019) 
398

399

400

401

402

403

404

405

406

407

408

409

410

411

412

413

414

415

416

417

418

419

420

421

422

423

424

425

426

427

428

429

430

431

432

433

434

435

436

because it is very difficult to enforce such requests. As a result, the current practice of providing researchers with even very small tissue samples from permanently archived material for use in individual sequencing projects results in highly non-optimal use of limited archived resources. In the case of the University of Kansas herpetological collections, we are increasingly finding that popular tissue samples have been nearly or completely exhausted after providing multiple prior tissue gifts to researchers. In many cases, these researchers sequenced only one or a few loci via Sanger sequencing, meaning that we provided them with orders of magnitudes more irreplaceable genetic material than was necessary for their work.

One possible solution to this extremely inefficient use of archived resources is to end the practice of providing researchers directly with subsamples of archived tissues and to instead provide researchers with only the amount of extracted DNA that is required for their particular application. For example, in the case of a project involving Sanger sequencing of one or two loci, a biodiversity archive could send the researchers 50-100 ng of extracted DNA instead of a destructively subsampled piece of tissue that is expected to yield 10,000 ng of DNA. Rather than resulting in researchers discarding large quantities of irreplaceable DNA, this practice would lead to archiving this material so that it could then fulfill subsequent requests for genetic material from the same specimen. However, this would require DNA extraction by biodiversity archive staff followed by quantification and provision of the appropriate amount of DNA for the researcher's required application. It would also require biodiversity collections to develop archival collections of not only tissues, but also extracted genomic DNA.

Although this approach could result in considerably more sustainable use of limited tissue resources, it does not come without substantial costs. First, it would require that staff at biodiversity collections extract and quantify DNA rather than merely sending a tissue sample. In many cases the staff responsible for preparing tissue loans will not have the requisite expertise, access to the necessary laboratory facilities, or time. Second, in-house extraction would require new protocols and facilities for archiving extracted DNA. Whether these costs are worthwhile will depend on the amount of material available and how heavily it is used by the research community. In the case of the University of Kansas herpetological collections, we now provide researchers only with an amount of extracted genomic DNA required for their research because we are finding that a significant number of samples in our archive have been used to the point that little or no tissue remains. We recommend that other biodiversity collections experiencing such over-use consider adopting a similar approach because it will radically improve sustainable use of genetic resources.

\section{Acknowledgements}

We thank Luke Welton for his assistance in accessing the University of Kansas herpetological collections. We thank the following curators and collections managers for their personal 
437 communications: Chris Huddleston, Lauren Scheinberg, Carol Spencer, and Breda Zimkus. We 438 thank Shea Lambert for his participation in Experiment 1.

439

440

441

442

443

444

445

446

447

448

449

450

451

452

453

454

455

456

457

458

459

460

461

462

463

464

465

466

467

468

469

470

471

472

473

474

475

\section{References}

Abdel-Latif A, Osman G. (2017). Comparison of three genomic DNA extraction methods to obtain high DNA quality from maize. Plant Methods 13-1.

Aguirre-Peñafiel V, Torres-Carvajal O, Sales Nunes P, Peck M, Maddock S. (2014). A new species of Riama Gray, 1858 (Squamata: Gymnophthalmidae) from the Tropical Andes. Zootaxa 3866-2:246-260.

Arbor Biosciences. (2019). myBaits ${ }^{\circledR}$ custom kits provide focused NGS hybridization capture panels for any organism and any project size.

Burrell A, Disotell T, Bergey C. (2015). The use of museum specimens with high-throughput DNA sequencers. Journal of Human Evolution 79:35-44.

Chambers EA, Hebert P. (2016). Assessing DNA Barcodes for Species Identification in North American Reptiles and Amphibians in Natural History Collections. PLoS ONE 114:e0154363.

Choi J, Lee H, Shipunov A. (2015). All that is gold does not glitter? Age, taxonomy, and ancient plant DNA quality. PeerJ 3:e1087.

Drabkova L, Kirschner J, Vlcek C. (2002). Comparison of seven DNA extraction and amplification protocols in historical herbarium specimens of Juncaceae. Plant Molecular Biology Reporter 20:161-175.

Droege G, Barker K, Astrin J, Bartels P, Butler C, Cantrill D, Coddington J, Forest F, Gemeinholzer B, Hobern D, Mackenzie-Dodds J, Ó Tuama É, Petersen G, Sanjur O, Schindel D, Seberg O. (2014). The Global Genome Biodiversity Network (GGBN) data portal. Nucleic Acids Research 42-D1:D607-D612.

Guo W, Jiang L, Bhasin S, Khan S, Swerdlow R. (2009). DNA extraction procedures meaningfully influence qPCR-based mtDNA copy number determination. Mitochondrion 9:261-265.

Hajibabaei M, deWaard J, Ivanova N, Ratnasingham S, Dooh R, Kirk S, Mackie P, Hebert P. (2005). Critical factors for assembling a high volume of DNA barcodes. Philosophical Transactions of the Royal Society B: Biological Sciences 360:1959-1967.

Hutter C.R., Cobb K.A., Portik, D., Travers S., Wood Jr. PL, and Brown, R.M. FrogCap: A modular sequence capture probe set for phylogenomics and population genetics for Anurans, assessed across multiple phylogenetic scales. bioRxiv 825307.

Hykin S, Bi K, McGuire J. (2015). Fixing Formalin: A method to recover genomic-scale DNA sequence data from formalin-fixed museum specimens using high-throughput sequencing. PLoS ONE 10-10:e0141579.

Jaksch K, Eschner A, Rintelen T, Haring E. (2016). DNA analysis of molluscs from a museum wet collection: a comparison of different extraction methods. BMC Research Notes. 
476 Kayes S, Cramp R, Hudson N, Franklin C. (2013). Effect of opioids on tissue metabolism in

477

478

479

480

481

482

483

484

485

486

487

488

489

490

491

492

493

494

495

496

497

498

499

500

501

502

503

504

505

506

507

508

509 aestivating and active green-striped burrowing frogs, Cyclorana alboguttata. Journal of Herpetology 47-2:369-377.

Kephart D, Krueger S, Grunst T, Shenoi H. (2006). A maximum instrument at a minimum size. Promega Notes 92:20-23.Naccarato A, Dejarnette J, Allman P. (2015). Successful establishment of a non-native species after an apparent single introduction event: investigating ND4 variability in introduced black spiny-tailed iguanas (Ctenosaura similis) in Southwestern Florida. Journal of Herpetology 49-2:230-236.

Promega. (2018). Maxwell(R) RSC Blood DNA Kit technical manual TM419. Promega Corporation.

Reineke A, Karlovsky P, Zebitz C. (1998). Preparation and purification of DNA from insects for AFLP analysis. Insect Molecular Biology 7-1:95-99.

Sawyer S, Krause J, Guschanski K, Savolainen V, Pääbo S. (2012). Temporal patterns of nucleotide misincorporations and DNA fragmentation in ancient DNA. PLoS ONE 73:e34131.

Schäffer S, Zachos F, Koblmüller S, Foo M, Agudelo N, Ranwez. (2017). Opening the treasure chest: a DNA-barcoding primer set for most higher taxa of Central European birds and mammals from museum collections. PLoS ONE 12-3:e0174449.

Schiebelhut L, Abboud S, Omez Daglio L, Swift H. (2016). A comparison of DNA extraction methods for high-throughput DNA analyses. Molecular Ecology Resources.

Stuart S, Chanson J, Cox N, Young B, Rodrigues A, Fischman D, Waller R. (2004). Status and trends of amphibian declines and extinctions worldwide. Science 306-5702:1783-1786.

Wang Z, Gerstein M, Snyder M. (2009). RNA-Seq: A revolutionary tool for transcriptomics. Nat. Rev. Genet. 10:57-63.

Watanabe M. (2015). The Nagoya Protocol on Access and Benefit Sharing: International treaty poses challenges for biological collections. BioScience 65-6:543-550.

Wilcox T, Zwickl D, Heath T, Hillis D. (2002). Phylogenetic relationships of the dwarf boas and a comparison of Bayesian and bootstrap measures of phylogenetic support. Molecular Phylogenetics and Evolution 25:361-371.

Yalçınkaya B, Yumbul E, Mozioğlu E, Akgoz M. (2017). Comparison of DNA extraction methods for meat analysis. Food Chemistry 221:1253-1257.

Zimkus B, Ford L. (2014). Genetic resource collections associated with natural history museums: a survey and analysis to establish a benchmark of standards. DNA Banking for the $21 \mathrm{st}$ Century. Proceedings of the U.S. Workshop on DNA Banking. pages 9-44. 


\section{Figure 1}

Total DNA yield vs tissue mass in Experiment 1

Total DNA yield was positively correlated with tissue mass in Experiment 1. This experiment was designed to test the effectiveness of the "eyeball" method for obtaining tissues appropriate for extraction. Each point represents a single tissue subsample taken in Experiment 1. Tissues were sampled via coarse visual estimate, then massed. We used a Pearson's correlation test to find the relationship between sample mass and total DNA yield. The red line indicates the results of this test when all data points were considered $(t=5.2299$, $r=0.7600, d f=20, p$-value $<0.001$ ), while the blue line indicates the results when the three greatest masses were excluded $(t=2.3112, r=0.4890, d f=17, p$-value $=0.0336)$. 


\section{Total DNA yield vs tissue mass in Experiment 1}

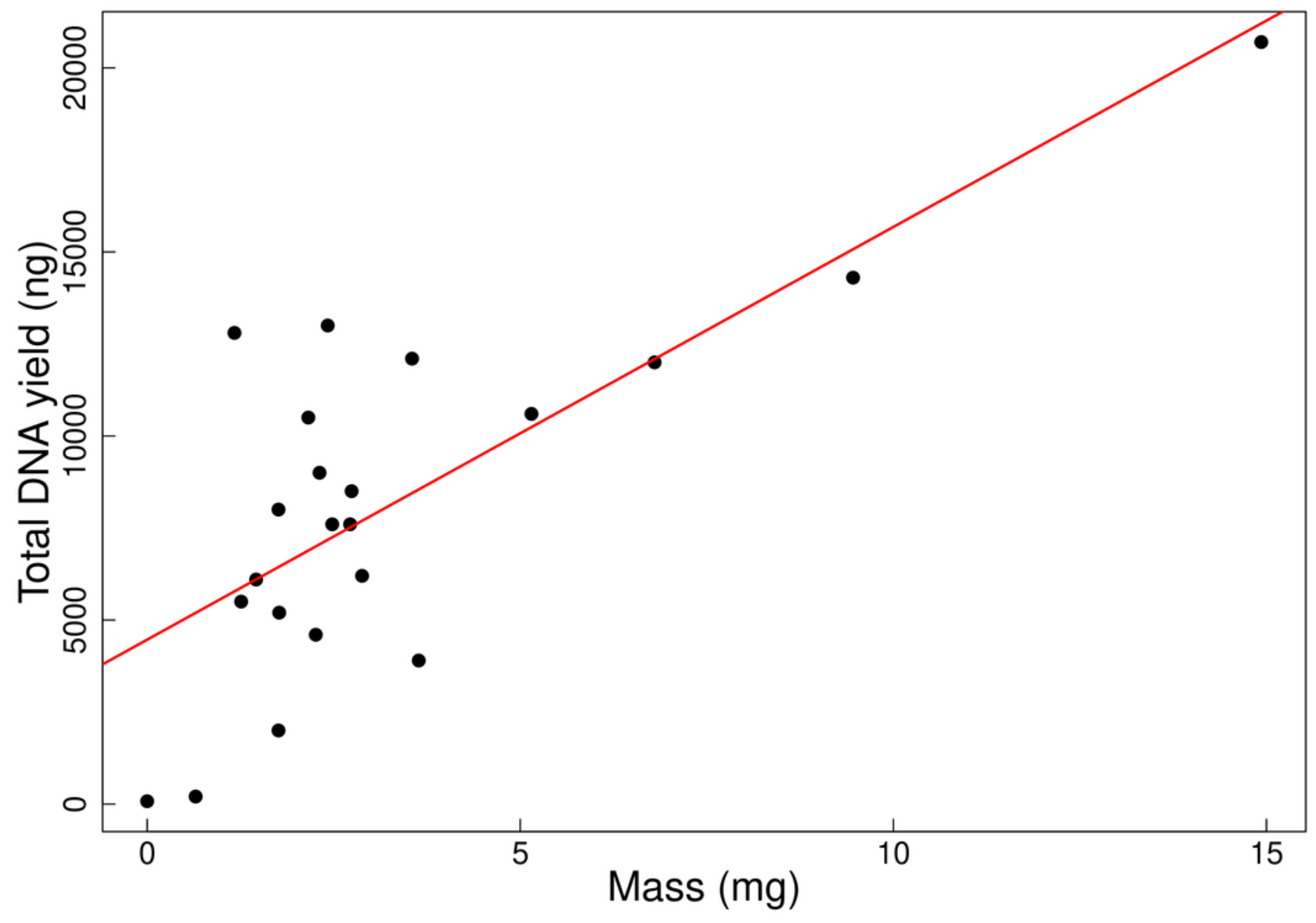




\section{Figure 2}

DNA yield vs tissue mass in Experiment 2

DNA yield was logarithmically correlated with tissue mass in Experiment 2. This experiment was designed to identify an optimal tissue mass for effective and efficient extraction. Each data point represents an individual tissue subsample taken at one of nine target masses: $1 \mathrm{mg}, 2 \mathrm{mg}, 4 \mathrm{mg}, 8 \mathrm{mg}, 10 \mathrm{mg}, 12 \mathrm{mg}, 16 \mathrm{mg}$, or $20 \mathrm{mg}$. The red line shows the trend in total DNA yield across the various masses tested $(y=3317.2 * \ln (x)+5030.3, R 2=0.29, p$-value $<0.0001$ ), while the blue line shows the trend in DNA yield per unit mass $(y=-1337 * \ln (x)+4470.3, R 2=0.55, p$-value $<0.0001)$. Trendlines were calculated using a least squares regression. 


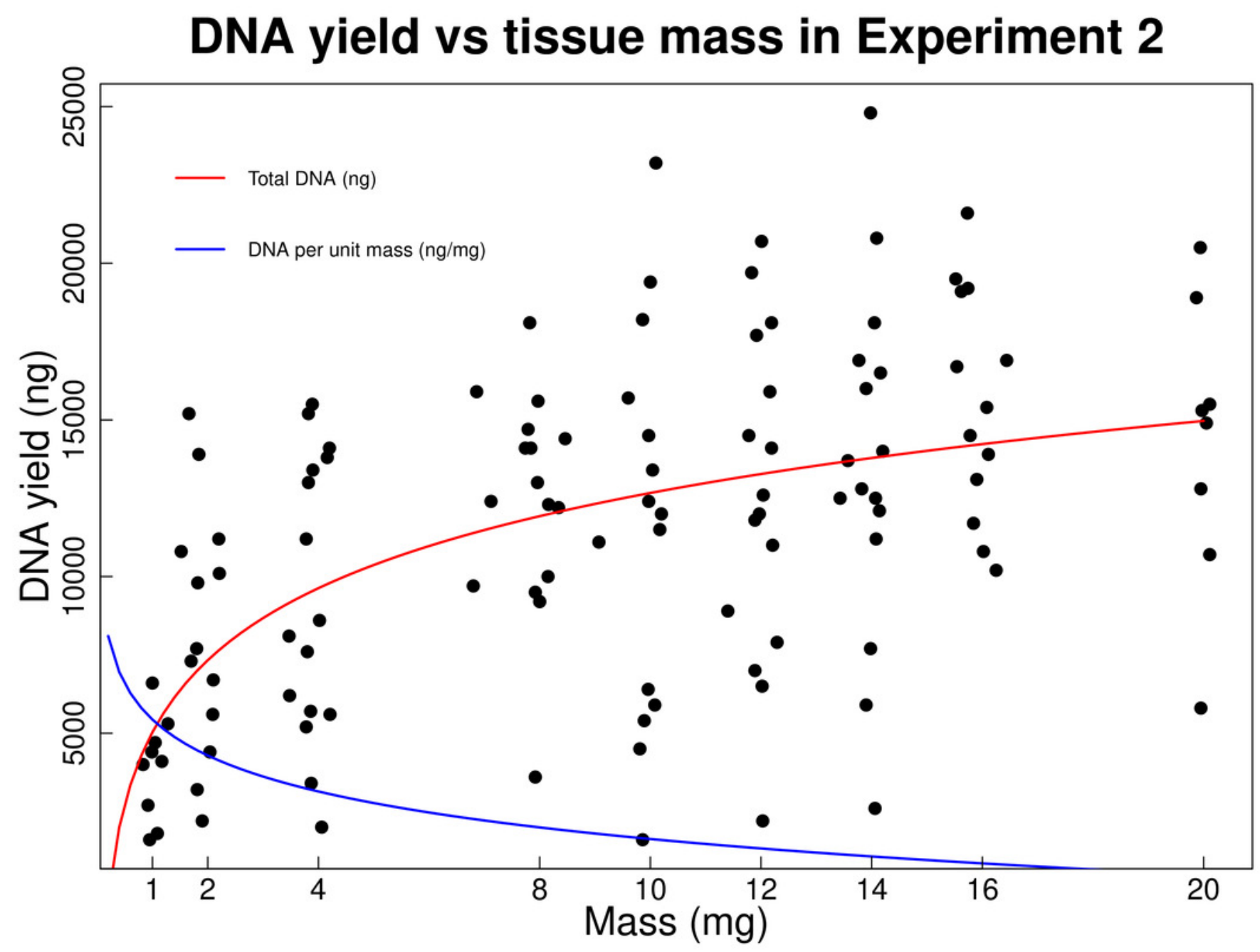


Figure 3

DNA yield vs tissue age in Experiment 4

DNA yield was not correlated with tissue age in Experiment 4. This experiment was designed to test the impact of tissue age on extractions when using an optimal mass. Each data point represents an individual tissue subsample of approximately $8 \mathrm{mg}$. The red line shows the relationship between the year the tissue was collected and the total DNA yield as calculated using a Pearson's correlation test, $r=-0.06, p$-value $=0.6904$.

DNA yield vs tissue age in Experiment 4

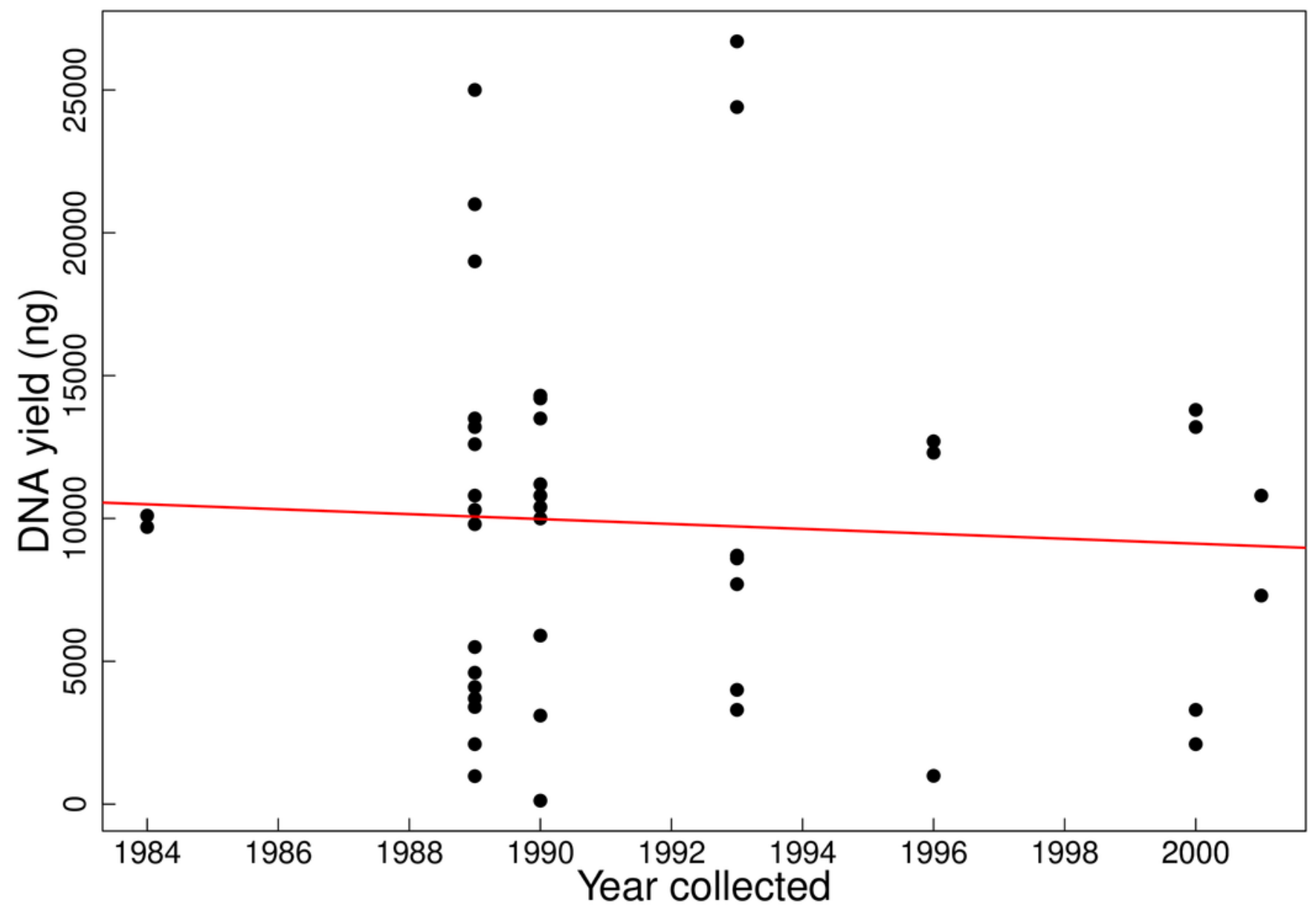

